Sebastiaan A. Bartels

Rick Bezemer

Floris J. Wallis de Vries

Dan M. J. Milstein

Alexandre Lima

Thomas G. V. Cherpanath

Anton H. van den Meiracker

Jasper van Bommel

Michal Heger

John M. Karemaker

Can Ince

\title{
Multi-site and multi-depth near-infrared
spectroscopy in a model of simulated (central) \\ Multi-site and multi-depth near-infrared
spectroscopy in a model of simulated (central) hypovolemia: lower body negative pressure
}

Received: 30 August 2010

Accepted: 7 December 2010

Published online: 21 January 2011

(C) The Author(s) 2011. This article is published with open access at Springerlink.com

S. A. Bartels and R. Bezemer contributed equally to this work.

Electronic supplementary material The online version of this article (doi: 10.1007/s00134-010-2128-6) contains supplementary material, which is available to authorized users.

\section{S. A. Bartels $(\bullet) \cdot$ R. Bezemer •}

A. Lima · J. van Bommel · C. Ince Department of Intensive Care,

Erasmus Medical Center, University

Medical Center, 's-Gravendijkwal 230,

3015 CE Rotterdam, The Netherlands

e-mail: s.bartels@erasmusmc.nl

Tel.: +31-20-5662140

Fax: +31-20-6967004

R. Bezemer

e-mail: r.bezemer@amc.uva.nl

\section{A. Lima}

e-mail: a.pintolima@eramusmc.nl

J. van Bommel

e-mail: j.vanbommel@erasmusmc.nl

C. Ince

e-mail: c.ince@amc.uva.nl

S. A. Bartels - R. Bezemer ·

F. J. W. de Vries

D. M. J. Milstein - C. Ince

Department of Translational Physiology, Academic Medical Center,

University of Amsterdam, Meibergdreef 9, 1105 AZ Amsterdam, The Netherlands
F. J. W. de Vries

e-mail: f.j.wallisdevries@amc.uva.nl

D. M. J. Milstein

e-mail: d.m.milstein@amc.uva.nl

T. G. V. Cherpanath

Division of Cardiology, Academic Medical Center, University of Amsterdam, Meibergdreef 9, 1105 AZ Amsterdam, The Netherlands e-mail: t.g.cherpanath@amc.uva.nl

A. H. van den Meiracker

Division of Vascular Medicine and Pharmacology, Department of Internal Medicine, Erasmus Medical Center, University Medical Center, 's-Gravendijkwal 230, 3015 CE Rotterdam, The Netherlands

e-mail: a.vandenmeiracker@erasmusmc.nl

M. Heger

Department of Experimental Surgery, Academic Medical Center, University of Amsterdam, Meibergdreef 9, 1105 AZ Amsterdam, The Netherlands

e-mail: m.heger@amc.uva.nl

\section{J. M. Karemaker}

Heart Failure Research Center, Academic Medical Center, University of Amsterdam, Meibergdreef 9, 1105 AZ Amsterdam, The Netherlands

e-mail: j.m.karemaker@amc.uva.nl

\section{J. M. Karemaker}

Department of Systems Physiology, Academic Medical Center, University of Amsterdam, Meibergdreef 9, 1105 AZ Amsterdam, The Netherlands
Abstract Purpose: To test the hypothesis that the sensitivity of nearinfrared spectroscopy (NIRS) in reflecting the degree of (compensated) hypovolemia would be affected by the application site and probing depth. We simultaneously applied multi-site (thenar and forearm) and multi-depth (15-2.5 and 25-2.5 mm probe distance) NIRS in a model of simulated hypovolemia: lower body negative pressure (LBNP). Methods: The study group comprised 24 healthy male volunteers who were subjected to an LBNP protocol in which a baseline period of $30 \mathrm{~min}$ was followed by a step-wise manipulation of negative pressure in the following steps: $0,-20,-40,-60$, -80 and $-100 \mathrm{mmHg}$. Stroke volume and heart rate were measured using volume-clamp finger plethysmography. Two multi-depth NIRS devices were used to measure tissue oxygen saturation $(\mathrm{StO} 2)$ and tissue hemoglobin index (THI) continuously in the thenar and the forearm. To monitor the shift of blood volume towards the lower extremities, calf THI was measured by single-depth NIRS. Results: The main findings were that the application of LBNP resulted in a significant reduction in stroke volume which was accompanied by a reduction in forearm $\mathrm{StO} 2$ and THI. Conclusions: NIRS can be used to detect changes in $\mathrm{StO} 2$ and THI consequent upon central 
hypovolemia. Forearm NIRS measurements reflect hypovolemia more sensitively than thenar NIRS measurements. The sensitivity of these NIRS measurements does not depend on NIRS probing depth. The LBNPinduced shift in blood volume is reflected by a decreased THI in the forearm and an increased THI in the calf.

\section{Keywords}

Near-infrared spectroscopy .

Lower body negative pressure .

Hypovolemia - Tissue oxygenation .

Microcirculation ·

Tissue hemoglobin content

Abbreviations

CO Cardiac output

HR Heart rate
LBNP Lower body negative pressure

MAP Mean arterial pressure

NIRS Near-infrared spectroscopy

$\mathrm{StO} 2$ Tissue oxygen saturation

SV Stroke volume

SVR Systemic vascular resistance

TFC Thoracic fluid content

THI Total hemoglobin index

\section{Introduction}

Hypovolemia is a major complication occurring in numerous clinical scenarios involving civilian, combat and disaster field traumas, surgical injuries, and intensive care settings $[1,2]$. The reduction in stroke volume (SV) associated with hypovolemia is physiologically countered by compensatory mechanisms such as increasing heart rate (HR) and systemic vascular resistance (SVR) to prevent the consequent decrease in blood pressure and organ perfusion. When these mechanisms fail, however, blood pressure will fall and adequate perfusion of vital organs will be at risk. Hence, detection of hypovolemia before blood pressure falls is of prime importance to initiate and/or guide treatment strategies aimed at maintaining adequate cardiac output (CO) and blood pressure in the prevention of organ hypoperfusion $[3,4]$.

To this end, near-infrared spectroscopy (NIRS) has been widely explored, successfully and unsuccessfully, for measuring tissue oxygen saturation $(\mathrm{StO} 2)$ and an index of tissue hemoglobin content (THI) in attempts to identify the presence and severity of hypovolemia [5-10]. However, the main problem with the interpretation of NIRS data from these studies is the diversity of methodologies used for the assessment of $\mathrm{StO} 2$ and THI. Two critical aspects concerning the methodology have been identified: the application site and the probing depth [7, 10]. The application site is important as differences may exist in the sensitivity of underlying muscle groups and other tissues to cardiovascular challenges such as central hypovolemia. In addition, the probing depth, which correlates directly with the NIRS probe size, determines the relative contribution of muscular and (sub)dermal tissue to the NIRS measurement $[7,10]$.

In an attempt to evaluate the detection of hypovolemia using NIRS, NIRS has recently been employed in a lower body negative pressure (LBNP) model of simulated central hypovolemia. Thenar $\mathrm{StO} 2$ was measured using a second-derivative NIRS device equipped with a $15-\mathrm{mm}$ probe and forearm $\mathrm{StO} 2$ was measured using a multidepth NIRS device equipped with a $30-\mathrm{mm}$ probe [8]. No change in $\mathrm{StO} 2$ in the thenar was found, while forearm $\mathrm{StO} 2$ significantly decreased. It was suggested that the forearm is a better application site for the detection of hypovolemia than the thenar and that the $15-\mathrm{mm}$ probe size is too small to collect light from a sufficiently deep layer of muscular tissue and thus is not able to detect hypovolemia. We criticized the applied methodology because the thenar and forearm measurements were performed in separate experiments rather than simultaneously and because the study did not investigate the effects of the $15-\mathrm{mm}$ probe on the forearm nor the effects of the 30-mm probe on the thenar [12]. Consequently, whether the superior detection of hypovolemia in the forearm was due to a more sensitive application site or due to greater probing depth remained inconclusive.

The aim of the present study was to test the hypothesis that the sensitivity of NIRS to reflect the degree of (compensated) hypovolemia would be affected by the application site and probing depth. For this purpose, we simultaneously applied multi-site (thenar and forearm) and multi-depth (15-mm and 25-mm probe) NIRS during LBNP as a model of controlled central hypovolemia.

\section{Materials and methods}

Subjects

The study participants comprised 24 healthy male volunteers. The study guidelines and procedures were reviewed and approved by the Medical Ethics Committee of the Academic Medical Center of the University of Amsterdam and voluntary written informed consent was obtained from all subjects. All participants were screened by a cardiologist, underwent exercise electrocardiography, and completed a medical history questionnaire prior to inclusion. All subjects were instructed to refrain from caffeine intake and other autonomic stimulants and to abstain from excessive physical work for at least $8 \mathrm{~h}$ prior to the LBNP experiment. 


\section{LBNP protocol and measurements}

A more detailed description of the materials and methods for the LBNP protocol and measurements can be found in the electronic supplementary material. In brief, the LBNP protocol consisted of a baseline period of $30 \mathrm{~min}$ followed by step-wise manipulation of negative pressure in the following steps: 0 to $-20,-40,-60,-80$, and -100 $\mathrm{mmHg}$. The LBNP protocol could be immediately terminated by the subject or attending physician in case of impending cardiovascular collapse or at the request of the subject. Cardiovascular and NIRS parameters were recorded at the end of each LBNP step and just before the onset of cardiovascular collapse. To monitor the shift of blood volume away from the upper body, thoracic bioimpedance (NICOM; Cheetah Medical, Wilmington, DE) was used to measure thoracic fluid content (TFC) which is expected to decrease when blood is shifted away from the upper body [13-15]. Additionally, to monitor the shift of blood volume towards the lower body, a single-depth 15-mm NIRS probe (InSpectra; Hutchinson Technology, Hutchinson, MN) was placed on the medial soleus of the left calf to monitor the calf THI which is expected to increase when blood is shifted towards the lower body [9]. To monitor the physiological changes associated with LBNP, CO, SV, HR, mean arterial pressure (MAP) and SVR were continuously and noninvasively measured using volume-clamp finger plethysmography (Nexfin monitor; BMEYE, Amsterdam, The Netherlands) [1618]. Two multi-depth NIRS devices (InSpectra) were used to measure $\mathrm{StO} 2$ and THI continuously and noninvasively in the left thenar eminence and in the lateral side of the anterior surface of the left forearm, as described previously $[11,21]$. Calculation of both the $\mathrm{StO} 2$ and the THI from the NIRS signal has been validated by Myers et al. [19-23]. We refer to the NIRS measurements on the forearm and thenar with the 15- $\mathrm{mm}$ and 25-mm probe spacing as $\mathrm{F}_{15}, \mathrm{~F}_{25}, \mathrm{~T}_{15}$ and $\mathrm{T}_{25}$, respectively.

\section{Statistical analysis}

Data plotting and analysis were performed in GraphPad Prism software (GraphPad Software, San Diego, CA). Data are presented as means \pm standard error of the means after the normal distribution of the data was confirmed by D'Agostino and Pearson normality tests. First, TFC, calf THI, and SV were plotted versus the level of LBNP, and statistical significance of differences between LBNP levels was analyzed using a one-way analysis of variance (ANOVA) for repeated measurements with a Bonferroni post-hoc test. $P<0.05$ was considered statistically significant. Second, all data were normalized and expressed as percentage of the baseline values. As the decrease in SV consequent to LBNP is the primary stimulus for physiological compensatory mechanisms to be activated (e.g. increased HR and SVR), the data were plotted versus SV (\% of baseline) and, to allow statistical analysis using ANOVA, data were categorized according to the following predefined categories: SV $=100 \%$ of baseline, SV $=100-80 \%$ of baseline, SV $=80-60 \%$ of baseline, and SV $=60-40 \%$ of baseline. Furthermore, Pearson's correlation analysis was performed for forearm $\mathrm{StO} 2$ (\% of baseline) and THI (\% of baseline) versus SV (\% of baseline).

\section{Results}

\section{Subject characteristics}

Since the tolerance of subjects to LBNP and the extent of the cardiovascular responses depend, amongst other factors, on age, physical fitness, gender, body size, and hydration status [24-29], a homogeneous subject population was recruited for this study. The study population consisted of 24 male subjects with an age of $28 \pm 1$ years, body weight of $82 \pm 2 \mathrm{~kg}$, and height of $182 \pm 1 \mathrm{~cm}$. A more detailed description of the results can be found in the electronic supplementary material.

LBNP-induced blood volume shift and stroke volume reduction

All 24 subjects completed the LBNP -20 and $-40 \mathrm{mmHg}$ steps, 23 subjects completed the LBNP $-60 \mathrm{mmHg}$ step, 15 subjects completed the LBNP $-80 \mathrm{mmHg}$ step, and 9 subjects completed the LBNP $-100 \mathrm{mmHg}$ step. Figure 1 shows that in all subjects, TFC decreased significantly $(P<0.05)$ and calf THI increased significantly $(P<0.05)$ with increasing LBNP. The decrease in TFC was associated with a significant decrease in SV $(P<0.05)$. As the decrease in SV consequent to LBNP was the primary trigger for compensatory mechanism activation, data were categorized according to $\mathrm{SV}=100 \%$ of baseline $(n=24), \mathrm{SV}=$ $100-80 \%$ of baseline $(n=36), \mathrm{SV}=80-60 \%$ of baseline $(n=34)$, and SV $=60-40 \%(n=25)$.

\section{Hemodynamic response}

In response to the decrease in SV, HR and SVR increased. With mild central hypovolemia induced by LBNP, HR remained unchanged $(P>0.05)$, but when hypovolemia progressed, HR increased significantly $(P<0.05)$. SVR increased significantly $(P<0.05)$ from baseline to mild central hypovolemia, but remained unchanged $(P>0.05)$ when SV decreased further. 

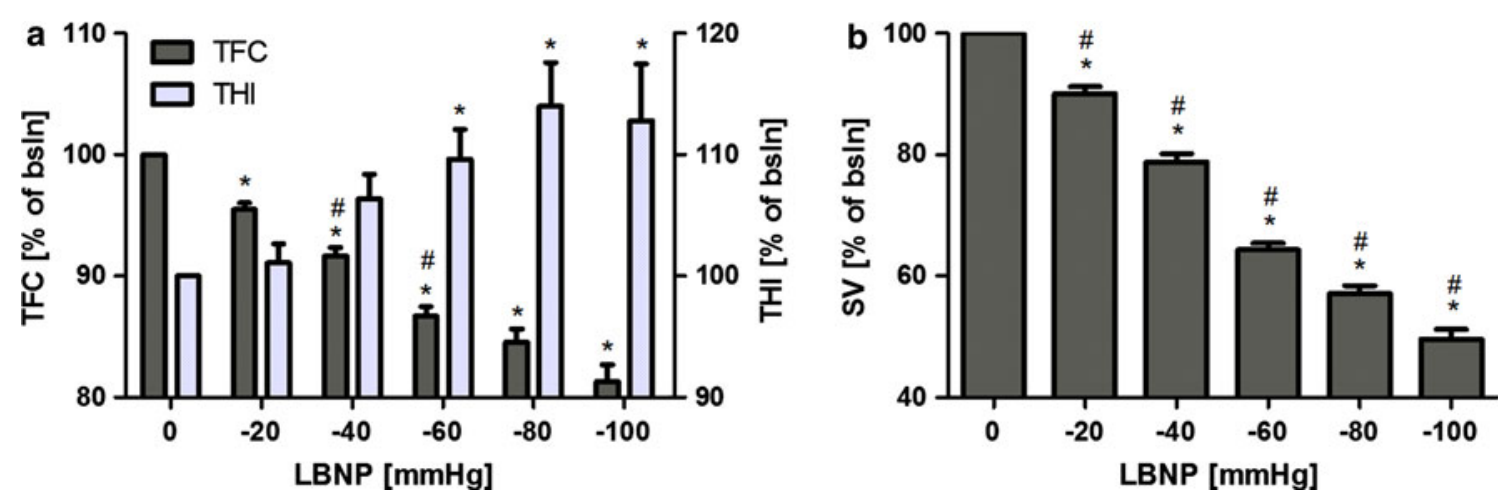

Fig. 1 a Thoracic fluid content (TFC) and calf tissue hemoglobin index (THI) and b stroke volume (SV) in relation to lower body negative pressure (LBNP), normalized and expressed as percentage

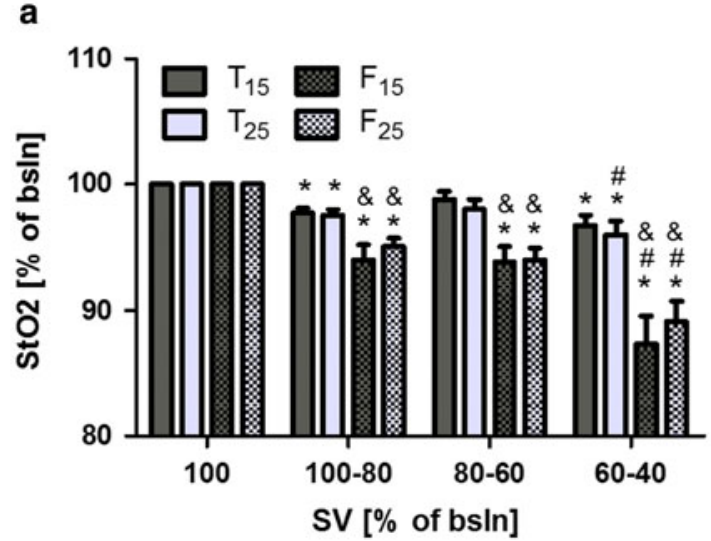

Fig. 2 a Thenar and forearm tissue oxygen saturation (StO2) and b thenar and forearm tissue hemoglobin index (THI) plotted versus stroke volume (SV). All parameters were normalized and expressed as percentage of baseline $(b \sin ) . \mathrm{T}_{15}, \mathrm{~T}_{25}, \mathrm{~F}_{15}$ and $\mathrm{F}_{25}$ are the measurements performed on the thenar and forearm using the

During the entire LBNP protocol, with exclusion of cardiovascular collapse, $\mathrm{CO}$ and MAP remained around the baseline levels $(P>0.05)$.

\section{Multi-site and multi-depth NIRS}

All NIRS results, expressed as percentage of baseline values, are shown in Fig. 2. In general, forearm NIRS measurements reflected changes in SV more sensitively than thenar NIRS measurements, and the sensitivity of these measurements did not depend on the NIRS probing depth. Whereas HR did not show a significant change in the first SV category of SV $=100-80 \%$ of baseline, forearm and thenar $\mathrm{StO} 2$ and $\mathrm{THI}$ did for both probing depths. However, measurements on the thenar showed only marginal changes. of baseline $(b \sin ) .{ }^{*} P<0.05$ versus LBNP $0 \mathrm{mmHg},{ }^{\#} P<0.05$ versus previous LBNP step

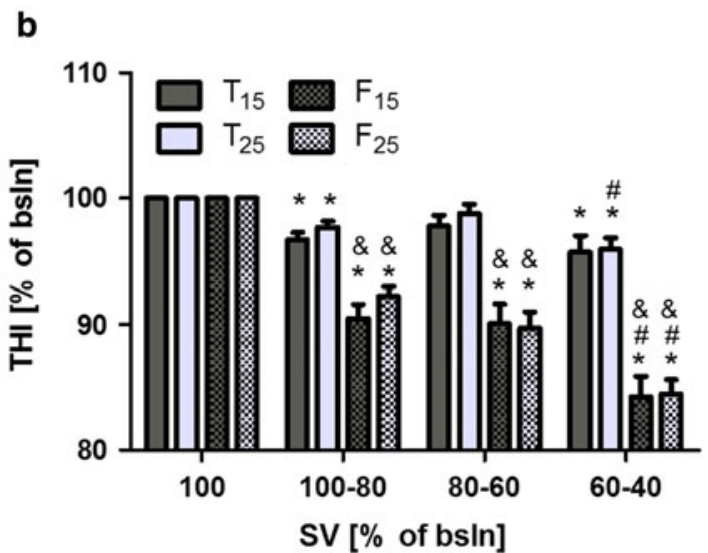

15-mm and 25 -mm probe spacing, respectively. $* P<0.05$ versus $\mathrm{SV}=100 \%$ of baseline, ${ }^{\#} P<0.05$ versus one SV category higher, and ${ }^{\&} P<0.05$ versus same probe on the thenar. $P>0.05$ for 15 versus $25 \mathrm{~mm}$

\section{Discussion and conclusions}

In the present study we simultaneously applied multi-site and multi-depth NIRS to test the hypothesis that the sensitivity of NIRS in reflecting the degree of (compensated) hypovolemia would be affected by the application site and probing depth. The main findings were that (1) the application of LBNP resulted in a significant shift in blood volume from the upper body to the lower body accompanied by a reduction in $\mathrm{SV},(2)$ this $\mathrm{SV}$ reduction consequent to LBNP was countered by an increase in HR and SVR, which adequately compensated for the decrease in SV and maintaining CO and MAP near baseline level, (3) NIRS can be used to detect changes in peripheral tissue oxygenation ( $\mathrm{StO} 2)$ and hemoglobin content (THI) resulting from central hypovolemia, (4) forearm NIRS measurements are more sensitive to LBNP-induced 
hypovolemia than thenar NIRS measurements, (5) the sensitivity of these NIRS measurements does not depend on NIRS probing depth, and (6) the LBNP-induced shift in blood volume from the upper body to the lower body is reflected by a decrease in THI in the forearm and an increase in THI in the calf.

To compensate for the central hypovolemia induced by LBNP, both HR and SVR increased. Initially, with mild central hypovolemia (i.e. SV $=100-80 \%$ of baseline), SVR increased by approximately $10 \%$ while HR remained unaffected. Then, with further reduction in SV (i.e. SV $=<80 \%$ of baseline), HR increased while SVR remained elevated around $10 \%$ above baseline level. Hence, we showed that compensation for mild hypovolemia is a biphasic response in which the SVR immediately increases followed by an increase in HR. As HR does not respond immediately to a reduction in SV and SVR cannot compensate during further reduction of $\mathrm{SV}$, these parameters are suboptimal for monitoring the onset and progression of hypovolemia.

In general, the increase in HR and SVR compensated adequately for the decrease in SV and maintained $\mathrm{CO}$ and MAP around baseline levels during the entire LBNP protocol. It must be noted, however, that cardiovascular collapse was not included in the analysis since the aim of the present study was to integratively investigate the sensitivity of NIRS in reflecting the degree of compensated hypovolemia during LBNP. Thus, in this early stage of hypovolemia, $\mathrm{CO}$ and MAP were maintained near the baseline levels, but by definition, at the moment of collapse, both CO and MAP rapidly decreased (data not shown).

Previous studies have demonstrated that during LBNP blood flow in the peripheral regions of the upper body decreases due to vasoconstriction induced by augmented muscle sympathetic nerve activity [30] and cardiopulmonary receptor unloading [31]. This is supported by our measurement of increased SVR during LBNP. Hansen et al. and Hachiya et al. have shown that this LBNPinduced (upper) body peripheral vasoconstriction is reflected by forearm NIRS measurements using a 30-mm NIRS probe size [9, 32]. Moreover, Fadel et al. showed that during LBNP forearm NIRS measurements using a $20 \mathrm{~mm}$ NIRS probe size are highly correlated with forearm blood flow velocity measured by Doppler ultrasound [33]. Reduced forearm blood flow and reduced THI result in greater microcirculatory oxygen extraction and thereby reduced forearm StO2 [34]. In summary, it is well established that central hypovolemia leads to (upper) body peripheral vasoconstriction and that hypovolemia is reflected by forearm NIRS measurements. Therefore, NIRS might be suitable for monitoring the volume status of intensive care patients or (post)surgical patients.

In previous studies, NIRS has been used to identify hypoperfusion, to predict organ dysfunction, and to guide resuscitation in trauma patients [35]. Crookes et al. [36] showed that thenar $\mathrm{StO} 2$ can reflect severe hypovolemic shock, but cannot identify mild or moderate shock. Cohn et al. reported no differences in thenar $\mathrm{StO} 2$ values between trauma patients and healthy volunteers. For this reason, NIRS cannot identify hypovolemia directly by assessment of steady-state (or baseline) StO2. However, these measurements were all carried out on thenar and no THI values were reported $[35,36]$.

Previously, we have found that forearm $\mathrm{StO} 2$ is a more sensitive parameter for detecting hypovolemia-induced peripheral vasoconstriction compared to thenar $\mathrm{StO} 2$ [11]. This LBNP study confirmed and strengthened our earlier findings. First, in a pilot study, we found that forearm $\mathrm{StO} 2$ was more responsive to head-up tilt than thenar $\mathrm{StO} 2$ [12]. Second, in a simple study on multi-depth thenar and forearm $\mathrm{StO} 2$ before and after a posture change, forearm $\mathrm{StO} 2$ was more responsive to the hemodynamic changes associated with the posture change than thenar StO2, independent of NIRS probing depth, which confirmed the findings of the pilot study [11]. Here we measured StO2 and THI with two multi-depth NIRS devices on the forearm and thenar simultaneously and again found that forearm $\mathrm{StO} 2$ and THI were more sensitive to central hypovolemia than thenar StO2 and THI. Although the marginal decrease (2-3\%) from baseline to SV $=60-40 \%$ of baseline in thenar $\mathrm{StO} 2$ was statistically significant, this finding is not (clinically) relevant. Forearm StO2, on the other hand, did decrease notably (8-9\%) and was similar to the decrease in forearm $\mathrm{StO} 2$ observed previously (11\% at a SV of about $40 \%$ of baseline) [7, 8]. Hence, the thenar and forearm NIRS measurements in the present study are comparable to those reported previously [7, 8], even though different NIRS devices were used. However, as this was the first study measuring $\mathrm{StO} 2$ and THI simultaneously in the thenar and forearm at multiple depths, we have now established that the sensitivity of the NIRS measurements is not dependent on probing depth but rather depends on measurement site. This finding rebuts the hypothesis postulated previously that the $15-\mathrm{mm}$ probe size on the forearm would be too small to reflect hypovolemia [8].

A potential physiological explanation for the difference between the thenar and forearm in terms of sensitivity to hypovolemia is that the circulation in the hands might be well-preserved during cardiovascular challenges. An example of such a phenomenon is coldinduced vasodilation during severe hypothermia, which opens the (micro)circulation to the hands in order to prevent cold-induced necrosis and thereby increase the chance of survival [37]. However, whether such a response also exists in hypovolemia remains unknown. Either way, although in the present study we could not identify why the forearm microcirculation was more sensitive to changes in volume status than the thenar microcirculation, we clearly showed that this is indeed the case and that the forearm is a more appropriate measurement site for NIRS for monitoring changes in the 
peripheral microcirculation in response to changes in volume status.

It should be noted, however, that there are some practical considerations regarding the use of NIRS in critically ill patients and the extrapolation of the results we present here to clinical scenarios. First, in the present study, no severe hypovolemia with hypotension or shock was included. However, the main objective was to use NIRS to detect hypovolemia in the compensated phase (i.e. with maintained blood pressure) and to test the hypothesis that the sensitivity of NIRS in reflecting the degree of (compensated) hypovolemia would be affected by the application site and probing depth. This we clearly showed, as differences between NIRS probing sites could be detected in this early, compensated phase of central hypovolemia. Second, it is important to realize that the NIRS measurements reflect changes in peripheral microcirculatory perfusion and that in the present study, these changes were induced by application of LBNP. We anticipate that vasoactive drug-related changes in peripheral microcirculatory perfusion would also be detected. However, when patients are on vasoactive drugs that prevent the peripheral microcirculation responding to changes in their volume status, NIRS would obviously not be able to detect hypovolemia as no changes would occur in the peripheral microcirculation. Third, another aspect potentially affecting the detection of hypovolemia using NIRS is peripheral edema. During LBNP, no peripheral edema was present. Still, the LBNP-related central hypovolemia is comparable to fluid loss-related hypovolemia as this type of hypovolemia would also decrease SV and lead to the activation of compensatory mechanisms such as increased HR and SVR. Whether the NIRS measurements would be able to reflect the degree of hypovolemia in the presence of peripheral edema, however, remains to be established, as tissue edema limits the oxygen offloading in the microcirculation and might thereby keep StO2 artificially high. The THI, in contrast, would probably decrease in fluid loss-related hypovolemia, as the THI is directly related to peripheral vascular tone. Fourth, other approaches, such as the assessment of SV variations or pulse pressure variations, might also provide information on changes in volume status. However, SV variations and pulse pressure variations both focus on the hypovolemia-related changes that occur on the arterial side of the circulation and therefore, in contrast to NIRS, do not reflect changes in the adequacy of tissue oxygenation that occur due to hypovolemia. Fifth, and finally, the changes observed in $\mathrm{StO} 2$ and $\mathrm{THI}$ were rather small (i.e. about $8-9 \%$ ) and might be difficult to detect in clinical practice. Therefore, whether the sensitivity of NIRS in detecting hypovolemia by could be increased by the addition of a dynamic test, such as a vascular occlusion test, should be explored [21].

In conclusion, the application of LBNP results in a significant blood volume shift and SV reduction that is countered by an increase in SVR and HR, which adequately compensate for the decrease in SV and maintain CO and MAP near baseline levels. NIRS can be used to detect central hypovolemia and applied on the forearm, NIRS more sensitively reflects changes in SV than when applied on the thenar. The sensitivity of NIRS does not depend on the NIRS probing depth. The present study confirmed the first part of the hypothesis that application site would affect the sensitivity of NIRS and rejected the second part of the hypothesis that probing depth would affect the sensitivity of NIRS.

Acknowledgments The NIRS devices used were provided by Hutchinson Technologies Inc. This study was in part supported by a grant from Hutchinson Technologies. Hutchinson Technologies had no part in the study design, data analysis, or drafting of the manuscript.

\section{Conflict of interest None.}

Open Access This article is distributed under the terms of the Creative Commons Attribution Noncommercial License which permits any noncommercial use, distribution, and reproduction in any medium, provided the original author(s) and source are credited.

\section{References}

1. Sauaia A, Moore FA, Moore EE, Moser KS, Brennan R, Read RA, Pons PT (1995) Epidemiology of trauma deaths: a reassessment. J Trauma 38:185-193

2. Bellamy RF (1984) The causes of death in conventional land warfare: implications for combat casualty care research. Mil Med 149:55-62

3. Orlinsky M, Shoemaker W, Reis ED, Kerstein MD (2001) Current controversies in shock and resuscitation. Surg Clin North Am 81:1217-1262
4. Wo CC, Shoemaker WC, Appel PL, Bishop MH, Kram HB, Hardin E (1993) Unreliability of blood pressure and heart rate to evaluate cardiac output in emergency resuscitation and critical illness. Crit Care Med 21:218-223

5. Jöbsis FF (1977) Noninvasive, infrared monitoring of cerebral and myocardial oxygen sufficiency and circulatory parameters. Science 198:1264-1267
6. Ward KR, Tiba MH, Ryan KL, Filho IP, Rickards CA, Witten T, Soller BR, Ludwig DA, Convertino VA (2010) Oxygen transport characterization of a human model of progressive hemorrhage. Resuscitation 81:987-993

7. Soller BR, Yang Y, Soyemi OO, Ryan KL, Rickards CA, Walz JM, Heard SO, Convertino VA (2008) Noninvasively determined muscle oxygen saturation is an early indicator of central hypovolemia in humans. J Appl Physiol 104:475-481 
8. Soller BR, Ryan KL, Rickards CA, Cooke WH, Yang Y, Soyemi OO, Crookes BA, Heard SO, Convertino VA (2008) Oxygen saturation determined from deep muscle, not thenar tissue, is an early indicator of central hypovolemia in humans. Crit Care Med 36:176-182

9. Hachiya T, Blaber AP, Saito M (2008) Near-infrared spectroscopy provides an index of blood flow and vasoconstriction in calf skeletal muscle during lower body negative pressure. Acta Physiol 193:117-127

10. Hachiya T, Blaber AP, Saito M (2004) Changes in superficial blood distribution in thigh muscle during LBNP assessed by NIRS. Aviat Space Environ Med 75:118-122

11. Bezemer R, Karemaker JM, Klijn E, Martin D, Mitchell K, Grocott M, Heger M, Ince C (2009) Simultaneous multi-depth assessment of tissue oxygen saturation in thenar and forearm using near-infrared spectroscopy during a simple cardiovascular challenge. Crit Care 19(Suppl 5):S5

12. Ince C, Bezemer R, Lima A (2009) Near infrared spectroscopy. Crit Care Med 37:384-385

13. Squara P, Rotcajg D, Denjean D, Estagnasie P, Brusset A (2009) Comparison of monitoring performance of Bioreactance vs. pulse contour during lung recruitment maneuvers. Crit Care 13:R125

14. Squara P, Denjean D, Estagnasie P, Brusset A, Dib JC, Dubois C (2007) Noninvasive cardiac output monitoring (NICOM): a clinical validation. Intensive Care Med 33:1191-1194

15. Raval NY, Squara P, Cleman M, Yalamanchili K, Winklmaier M, Burkhoff D (2008) Multicenter evaluation of noninvasive cardiac output measurement by bioreactance technique. J Clin Monit Comput 22:113-119

16. Wesseling KH, Settels JJ, van der Hoeven GM, Nijboer JA, Butijn MW, Dorlas JC (1985) Effects of peripheral vasoconstriction on the measurement of blood pressure in a finger. Cardiovasc Res 19:139-145

17. Parati G, Casadei R, Groppelli A, Di Rienzo M, Mancia G (1989) Comparison of finger and intra-arterial blood pressure monitoring at rest and during laboratory testing. Hypertension 13:647-655
18. Imholz BP, Settels JJ, van der Meiracker AH, Wesseling KH, Wieling W (1990) Non-invasive continuous finger blood pressure measurement during orthostatic stress compared to intra-arterial pressure. Cardiovasc Res 24:214-221

19. Myers DE, Anderson LD, Seifert RP, Ortner JP, Cooper CE, Beilman GJ, Mowlem JD (2005) Noninvasive method for measuring local hemoglobin oxygen saturation in tissue using wide gap second derivative near-infrared spectroscopy. J Biomed Opt 10:034017

20. Myers D, McGraw M, George M, Mulier K, Beilman G (2009) Tissue hemoglobin index: a non-invasive optical measure of total tissue hemoglobin. Crit Care 13(Suppl 5):S2

21. Bezemer R, Lima A, Myers D, Klijn E, Heger M, Goedhart PT, Bakker J, Ince C (2009) Assessment of tissue oxygen saturation during a vascular occlusion test using near-infrared spectroscopy: the role of probe spacing and measurement site studied in healthy volunteers. Crit Care 13(Suppl 5):S4

22. Chance B, Dait MT, Zhang C, Hamaoka T, Hagerman F (1992) Recovery from exercise-induced desaturation in the quadriceps muscles of elite competitive rowers. Am J Physiol 262:C766-C775

23. Cui W, Kumar C, Chance B (1991) Experimental study of migration depth for the photons measured at sample surface. Proc SPIE 1431:180-191

24. Cooke WH, Ryan KL, Convertino VA (2004) Lower body negative pressure as a model to study progression to acute hemorrhagic shock in humans. J Appl Physiol 96:1249-1261

25. Convertino VA, Ludwig DA, Cooke WH (2004) Stroke volume and sympathetic responses to lower-body negative pressure reveal new insight into circulatory shock in humans. Auton Neurosci 30(111):127-134

26. Convertino VA (2001) Lower body negative pressure as a tool for research in aerospace physiology and military medicine. J Gravit Physiol 8:1-14

27. Goswami N, Loeppky JA, HinghoferSzalkay H (2008) LBNP: past protocols and technical considerations for experimental design. Aviat Space Environ Med 79:459-471
28. Goswami N, Grasser E, Roessler A, Schneditz D, Hinghofer-Szalkay H (2009) The cardiovascular response to lower body negative pressure in humans depends on seal location. Physiol Res 58:311-318

29. Stevens PM, Lamb LE (1965) Effects of lower body negative pressure on the cardiovascular system. Am J Cardiol 16:506-515

30. Rea RF, Wallin BG (1989) Sympathetic nerve activity in arm and leg muscles during lower body negative pressure in humans. J Appl Physiol 66:2778-2781

31. Abboud FM, Eckberg DL, Johannsen UJ, Mark AL (1979) Carotid and cardiopulmonary baroreceptor control of splanchnic and forearm vascular resistance during venous pooling in man. J Physiol 286:173-184

32. Hansen J, Sander M, Hald CF, Victor RG, Thomas GD (2000) Metabolic modulation of sympathetic vasoconstriction in human skeletal muscle: role of tissue hypoxia. J Physiol 527(Pt 2):387-396

33. Fadel PJ, Keller DM, Watanabe H, Raven PB, Thomas GD (2004) Noninvasive assessment of sympathetic vasoconstriction in human and rodent skeletal muscle using near-infrared spectroscopy and Doppler ultrasound. J Appl Physiol 96:1323-1330

34. Skarda DE, Mulier KE, Myers DE, Taylor JH, Beilman GJ (2007) Dynamic near-infrared spectroscopy measurements in patients with severe sepsis. Shock 27:348-353

35. Cohn SM, Nathens AB, Moore FA, Rhee P, Puyana JC, Moore EE, Beilman GJ (2007) Tissue oxygen saturation predicts the development of organ dysfunction during traumatic shock resuscitation. J Trauma 62:44-55

36. Crookes BA, Cohn SM, Bloch S, Amortegui J, Manning R, Li P, Proctor MS, Hallal A, Blackbourne LH, Benjamin R, Soffer D, Habib F, Schulman CI, Duncan R, Proctor KG (2005) Can near-infrared spectroscopy identify the severity of shock in trauma patients? J Trauma 58:806-813

37. Daanen HA (2003) Finger cold-induced vasodilation: a review. Eur J Appl Physiol 89:411-426 